Open Access

\title{
Functional differences between microglia and monocytes after ischemic stroke
}

\author{
Rodney M. Ritzel ${ }^{1}$, Anita R. Patel ${ }^{1}$, Jeremy M. Grenier ${ }^{1}$, Joshua Crapser ${ }^{1}$, Rajkumar Verma', Evan R. Jellison² \\ and Louise D. McCullough ${ }^{1 *}$
}

\begin{abstract}
Background: The brain's initial innate response to stroke is primarily mediated by microglia, the resident macrophage of the CNS. However, as early as $4 \mathrm{~h}$ after stroke, the blood-brain barrier is compromised and monocyte infiltration occurs. The lack of discriminating markers between these two myeloid populations has led many studies to generate conclusions based on the grouping of these two populations. A growing body of evidence now supports the distinct roles played by microglia and monocytes in many disease models.
\end{abstract}

Methods: Using a flow cytometry approach, combined with ex-vivo functional assays, we were able to distinguish microglia from monocytes using the relative expression of CD45 and assess the function of each cell type following stroke over the course of 7 days.

Results: We found that at $72 \mathrm{~h}$ after a 90-min middle cerebral artery occlusion (MCAO), microglia populations decrease whereas monocytes significantly increase in the stroke brain compared to sham. After stroke, BRDU incorporation into monocytes in the bone marrow increased. After recruitment to the ischemic brain, these monocytes accounted for nearly all BRDU-positive macrophages. Inflammatory activity peaked at $72 \mathrm{~h}$. Microglia produced relatively higher reactive oxygen species and TNF, whereas monocytes were the predominant IL-1 $\beta$ producer. Although microglia showed enhanced phagocytic activity after stroke, monocytes had significantly higher phagocytic capacity at $72 \mathrm{~h}$. Interestingly, we found a positive correlation between TNF expression levels and phagocytic activity of microglia after stroke.

Conclusions: In summary, the resident microglia population is vulnerable to the effects of severe ischemia, show compromised cell cycle progression, and adopt a largely pro-inflammatory phenotype after stroke. Infiltrating monocytes are primarily involved with early debris clearance of dying cells. These findings suggest that the early wave of infiltrating monocytes may be beneficial to stroke repair and future therapies aimed at mitigating microglia cell death may prove more effective than attempting to elicit targeted anti-inflammatory responses from damaged cells.

Keywords: Microglia, Monocytes, Stroke, Inflammation, Phagocytosis

\section{Introduction}

Inflammation is a key component of stroke-induced injury and elevated levels of inflammatory markers are associated with poor outcome in stroke patients [1-4]. Inflammation in the brain is generally mediated by microglia, the resident macrophage of the CNS [5]. In the protected confines of the blood-brain barrier, microglia maintain healthy brain function by clearing debris, pruning synapses, and producing growth/repair factors

\footnotetext{
* Correspondence: Imccullough@uchc.edu

'Department of Neurology, University of Connecticut Health Center, 263

Farmington Avenue, Farmington, CT 06030, USA

Full list of author information is available at the end of the article
}

[6]. These cells stand poised to respond to injuries in the CNS such as ischemic stroke. Numerous experimental studies have shown that microglia become activated following stroke, notably shifting their morphology from a thin, ramified state to a large, amoeboid structure [7]. This change is generally thought to be accompanied by an increase in proliferation and production of inflammatory mediators. Yet despite the evidence for widespread recruitment of bone marrow-derived monocytes (and their derivatives) to injured brain regions and a lack of discriminate cell markers, little is known regarding the functional differences between these two myeloid populations in ischemic stroke. Given the high degree of 
macrophage heterogeneity that comprises our innate immune system, the functional role of these populations is likely distinct and of translational importance [8-11]. CNS-resident microglia are the first responders to ischemia; these cells likely serve a unique role in injury repair relative to monocytes, which have a finite lifespan and are recruited in higher numbers during the postreperfusion phase from the periphery. These differences have been made evident in recent studies that utilize transgenic bone marrow chimeras to distinguish between local and circulating myeloid populations [12]. These potentially disparate roles suggest that drugs designed to modulate microglia function may adversely impact that of the infiltrating monocyte population.

In this study, we investigated functional differences between brain-resident microglia and infiltrating monocytes in acute ischemic injury to better understand the contribution of each population to the recovery phase of stroke. Using flow cytometry to discriminate between these two populations, we applied ex-vivo functional assays to ascertain their functional roles during stroke and early recovery. By identifying the distinct function of microglia and monocytes early after ischemic stroke, the potential for targeting these specific cell populations will allow for the development of more effective therapeutic interventions.

\section{Materials and methods Mice/animals}

Young adult C57BL/6 J male mice (10-12 weeks) of age were pair-housed on sawdust bedding in a pathogen free facility (light cycle 12/12 h light/dark). All animals had access to chow and water ad libitum. All procedures were performed in accordance with NIH guidelines for the care and use of laboratory animals and approved by the Institutional Animal Care and Use Committee of the University of Connecticut Health Center. All analyses were performed blinded to surgical conditions.

\section{Ischemic stroke model}

Cerebral ischemia was induced by $90 \mathrm{~min}$ of reversible middle cerebral artery occlusion (MCAO, 20-25 gm mice) under isoflurane anesthesia as previously described [13]. Rectal temperatures were maintained at approximately $37{ }^{\circ} \mathrm{C}$ during surgery and ischemia with an automated temperature control feedback system. A midline ventral neck incision was made, and unilateral MCAO was performed by inserting a 6.0 Doccol monofilament (Doccol Corp, Redlands, CA, USA) into the right internal carotid artery $6 \mathrm{~mm}$ from the internal carotid/pterygopalatine artery bifurcation via an external carotid artery stump. Following reperfusion mice were sacrificed at 24 and $72 \mathrm{~h}$ and 7 days. Sham-operated animals underwent the same surgical procedure, but the suture was not advanced into the internal carotid artery.

\section{Tissue harvesting}

Mice were euthanized, transcardially perfused with $60 \mathrm{~mL}$ cold, sterile PBS, and the brains were harvested. The brainstem, cerebellum, and olfactory bulbs were removed. The brain was then divided along the interhemispheric fissure into two hemispheres and subsequently rinsed with PBS to remove contaminant cells.

\section{Flow cytometry}

Brains were placed in complete Roswell Park Memorial Institute (RPMI) 1640 (Lonza) medium and mechanically and enzymatically digested in collagenase/dispase $(1 \mathrm{mg} / \mathrm{mL})$ and DNAse $(10 \mathrm{mg} / \mathrm{mL}$; both Roche Diagnostics) for $1 \mathrm{~h}$ at $37{ }^{\circ} \mathrm{C}$. The cell suspension was filtered through a 70 um filter. Leukocytes were harvested from the interphase of a $70 \% / 30 \%$ Percoll gradient. Cells were washed and blocked with mouse Fc Block (eBioscience) prior to staining with primary antibody-conjugated flourophores: CD45-eF450, CD11b-APCeF780, Ly6C-PerCPCy5.5, Ly6G-PE, and SIRP $\alpha$-APC. All antibodies were commercially purchased from eBioscience. For live/dead discrimination, a fixable viability dye, carboxylic acid succinimidyl ester (CASE-AF350, Invitrogen), was diluted at 1:300 from a working stock of $0.3 \mathrm{mg} / \mathrm{mL}$. Cells were briefly fixed in $2 \%$ paraformaldehyde (PFA). Data were acquired on a LSRII using FACSDiva 6.0 (BD Biosciences) and analyzed using Flowjo (Treestar Inc.). No less than 100,000 events were recorded for each sample. Resident microglia were identified as the CD $45^{\text {int }}$ CD $11 b^{+}{\text {Ly } 6 C^{-}}^{-}$ population, whereas bone marrow-derived leukocytes were identified as CD $45^{\text {hi }}$ CD $11 b^{+}$Ly $_{6} C^{+}$Ly $6 G^{-}$. Cell typematched fluorescence minus one (FMO) controls were used to determine the positivity of each antibody. Prior to assessment on the cytometer, isolated cells were briefly probed to determine phagocytosis activity, oxidative stress level, cell proliferation status, and cytokine production as described below.

\section{Phagocytosis bead assay}

To study the phagocytic activity of microglia, fluorescent latex beads (Fluoresbrite Yellow Green (YG) carboxylate microspheres; 1um diameter; Polysciences) were added to sorted microglia in a final dilution of 1:100 as described [14]. After 1-h incubation at $37^{\circ} \mathrm{C}$, the cells were washed three times with FACS buffer, resuspended in FACS buffer, stained for surface markers, and fixed in PFA ( $N=6$ /group). Mean fluorescence was determined from the YG bead + microglia population and used to measure the amount of beads per phagocytosing cell [15]. 


\section{Reactive oxygen species measurement}

For detection of reactive oxygen species, microglial cells were incubated with redox-sensitive DHR (5uM; Ex/Em: 495/520) fluorogenic cell-permeant dye (Life Technologies, Invitrogen). Cells were incubated for $20 \mathrm{~min}$ at $37{ }^{\circ} \mathrm{C}$, washed three times with FACS buffer (without NaAz), and then stained for surface markers including CASE $(N=5$ / group). After loading cells with DHR, each sample was separated into two tubes, one kept on ice and one at $37^{\circ} \mathrm{C}$.

\section{BRDU labeling and analysis}

For cell proliferation studies, $50 \mathrm{mg} / \mathrm{kg}$ of BRDU (Sigma) was injected interperitoneally starting at $12 \mathrm{~h}$ after reperfusion and again at 24 and 48 h. BRDU staining was assessed using a BRDU Flow Kit (BD Biosciences). In brief, cells were permeabilized with detergent and treated with DNAse prior to the addition of both anti-BRDUFITC antibody and Ki67-PE (eBioscience).

\section{Intracellular cytokine production}

For intracellular cytokine staining, an in vivo brefeldin A (BFA) protocol was followed. Briefly, $10 \mathrm{~mL} / \mathrm{kg}$ of BFA (Sigma, $0.5 \mathrm{mg} / \mathrm{mL}$ in DMSO) was injected via tail vein. Ten hours later, animals were sacrificed and tissue was harvested as noted above. Prior to staining, $1 \mathrm{ul}$ of GolgiPlug containing brefeldin A (BD Biosciences) was added to $800 \mathrm{ul}$ complete RPMI and cells were incubated for $2 \mathrm{~h}$ at $37{ }^{\circ} \mathrm{C}$ (5\% CO2). Afterward, cells were re-suspended in Fc Block, stained for surface antigens and washed in $100 \mathrm{ul}$ of fixation/permeabilization solution (BD Biosciences) for $20 \mathrm{~min}$. Microglia were then washed twice in $300 \mathrm{ul}$ permeabilization/wash buffer (BD Biosciences), resuspended in an intracellular antibody cocktail containing TNF-PE-Cy7 (eBioscience) and IL-1 $\beta$-FITC (eBioscience) and subsequently fixed ( $N=5-7$ /group).

\section{Bone marrow chimera generation}

Wildtype C57BL/6 J mice (8 weeks old) were lethally irradiated (two doses of 5-6 Gy) in a Gammacell 40 research irradiator, and $5 \times 10^{5}$ nucleated GFP-expressing donor bone marrow cells were injected retro-orbitally [16]. Chimeras were maintained on sulfamethoxazole/trimethoprim antibiotics in their drinking water 1 day prior and 2 weeks following irradiation. Chimeras were used for experiments 10 weeks following transplantation.

\section{Statistical analyses}

Data from individual experiments are presented as mean \pm SEM and assessed by Student's $t$ test or one-way ANOVA with Tukey post-hoc test for multiple comparisons (GraphPad Prism Software Inc, San Diego, CA, USA). For two-way ANOVA, significant differences between paired comparisons were conducted with the Holm-Sidak test. The Spearman's rank correlation test was used to ascertain the correlation between cytokine production and phagocytic activity. Significance was set at $p<0.05$.

\section{Results}

Ischemic stroke induces microglial death, bone marrow production of monocytes, and recruitment of monocytes to the injured brain

We confirmed the ability to reliably distinguish CD $45^{\text {int }}$ microglia from CD $45^{\text {hi }}$ monocyte populations in the ischemic brain by generating GFP bone marrow chimeras, in which all bone marrow-derived cells were GFP-positive (Additional file 1: Figure S1A). We demonstrated that the two populations did not significantly overlap after stroke, validating this approach. Absolute leukocyte counts were obtained by flow cytometry at 24 and $72 \mathrm{~h}$ after MCAO in non-irradiated, intact wild type mice using the gating strategy shown in Fig. 1a, b. A significant reduction in the number of microglia $\left(\mathrm{CD} 45^{\text {int }} \mathrm{CD} 11 \mathrm{~b}^{+} \mathrm{Ly} 6 \mathrm{C}^{-}\right)$after stroke was found after $24 \mathrm{~h}(p<0.05$; Fig. 1c). Conversely, we found a dramatic increase in monocyte $\left(\mathrm{CD} 45^{\mathrm{hi}} \mathrm{CD} 11 \mathrm{~b}^{+} \mathrm{Ly}_{6 \mathrm{C}^{+}} \mathrm{Ly}_{6 \mathrm{G}^{-}}\right)$counts in the stroke hemisphere compared to sham brain (Fig. 1d). At 72 h, microglia expressed increased levels of Ki67, a marker of actively cycling cells $(p=0.017$; Fig. 2a, b). DNA synthesis, an indicator of cell proliferation, was then measured by BRDU incorporation. Following repeated BRDU injections starting at $12 \mathrm{~h}$, microglia showed little BRDU incorporation by $72 \mathrm{~h}$, whereas $\sim 90 \%$ of monocytes in the ischemic brain were BRDU-positive (Fig. 2c, d). Stroke is known to stimulate bone marrow production of myeloid cells that are subsequently recruited to the brain [17]. We found a significant increase in the percentage of $\mathrm{BRDU}^{+}$ monocytes in the bone marrow following stroke $(p=0.016$; Fig. 2e, f). These data suggest that after 90-min tMCAO, there is a significant loss of resident microglia, impaired cell cycle progression, and an increased number of newly produced, bone marrow-derived monocytes.

\section{Differential oxidative stress and cytokine production by microglia and monocytes after stroke}

Reactive oxygen species production drives the oxidative stress response to stroke and is critical to injury progression [18]. We examined free radical formation in microglia and monocytes after stroke. Resident microglia expressed significantly more ROS relative to the infiltrating monocytes $(p<0.01)$, as evidenced by increased DHR123 staining intensity (Fig. 3a, b). Because oxidative stress precipitates cytokine production, we next evaluated pro-inflammatory cytokine levels in these cells. Newly recruited monocytes expressed higher levels of TNF at 24 h; however, microglia expressed significantly greater levels by $72 \mathrm{~h}$ (Fig. 3c, d). Infiltrating monocytes expressed relatively higher levels of IL-1 $\beta$ at all time points $(p<0.001$; Fig. 3e, f). The number of microglia expressing TNF 

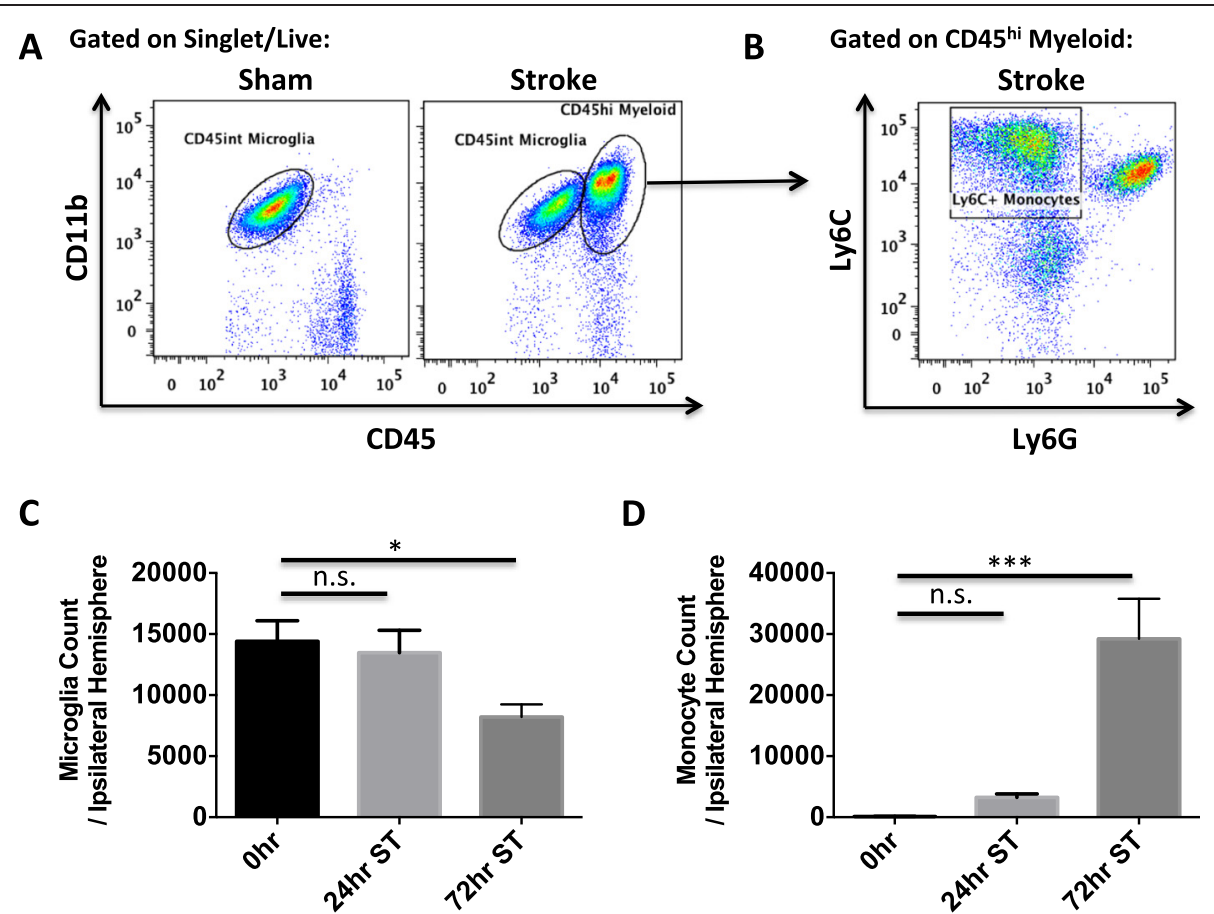

Fig. 1 Number of microglia and monocytes in the ischemic hemisphere in the early period after stroke. A representative dot plot depicts the gating strategy used to identify both brain-resident microglia and Ly6C $\mathrm{C}^{+}$monocytes at $72 \mathrm{~h}$ after $90-$ min MCAO (a, b). Absolute cell counts of microglia (c) and monocytes (d) were quantified at 0, 24, and $72 \mathrm{~h}$ after stroke. For all experiments, $N=9 /$ group. Error bars show mean SEM. Abbreviation: SEM standard error of the mean. ${ }^{*} p<0.05 ;{ }^{* * *} p<0.001$

remained elevated above sham levels at 7 days, whereas those expressing IL-1 $\beta$ did not differ. Neither microglia nor brain-recruited monocytes expressed detectable levels of the anti-inflammatory cytokines IL-4 and IL-10 after stroke (data not shown). These results suggest that microglia play a critical role in oxidative injury and that these two cell types may mediate cell death via distinct signaling pathways.

\section{Monocytes are the predominant phagocytes in the brain at $72 \mathrm{~h}$ after stroke}

Microglia in the ischemic hemisphere were significantly more activated based on side scatter (granularity) properties compared to sham, suggesting enhanced uptake of dying cells and debris (Fig. 4a, b). Phagocytosis is important in debris clearance and injury repair. Using a bead assay, we measured phagocytic activity of microglia and monocytes (Fig. 4c). After stroke, the percentage of microglia that phagocytosed beads significantly increased at $24 \mathrm{~h}(p<0.001)$ and peaked at a near four-fold increase by $72 \mathrm{~h}(p<0.01$; Fig. $4 \mathrm{~d})$. The phagocytic activity of microglia is restored back to baseline levels by 7 days. Compared to microglia, however, infiltrating monocytes exhibited far greater capacity for phagocytosis. Significantly more monocytes were bead-positive as were the number of beads phagocytosed per cell as evidenced by MFI ( $p<0.001$; Fig. 4e). Monocytes at $72 \mathrm{~h}$ also expressed significantly higher levels of the phagocytic marker SIRP $\alpha$ than did microglia ( $p=0.005$; Fig. 4f, g). Interestingly, phagocytic microglia were more likely to express TNF and at higher levels than non-phagocytic microglia, indicating that pro-inflammatory (M1) markers may overlap with anti-inflammatory (M2) function (Fig. 5a, b). Moreover, a positive correlation was found between the level of TNF production and the number of beads phagocytosed by microglia after stroke ( $p=0.0167$; Fig. $5 c$ ). Taken together, these data imply that microglia do increase phagocytic activity following stroke stimulus, albeit at significantly lower levels than recruited monocytes.

\section{Discussion}

We demonstrate for the first time that the increase in macrophage proliferation in the brain following severe tMCAO is a result of stimulus-driven bone marrow production of migrant monocytes rather than proliferation of microglia. Resident microglia, as bystanders to ischemia, are similarly vulnerable to its detrimental effects. We found that after a 90-min MCA occlusion, the number of microglia is significantly less than that seen in sham brain. Traditionally, the use of standard immunohistochemistry could not make this distinction; possibly overestimating the microglial response leads to the concept that microglia are highly proliferative, even after severe ischemic injuries. Indeed, the microglia population 


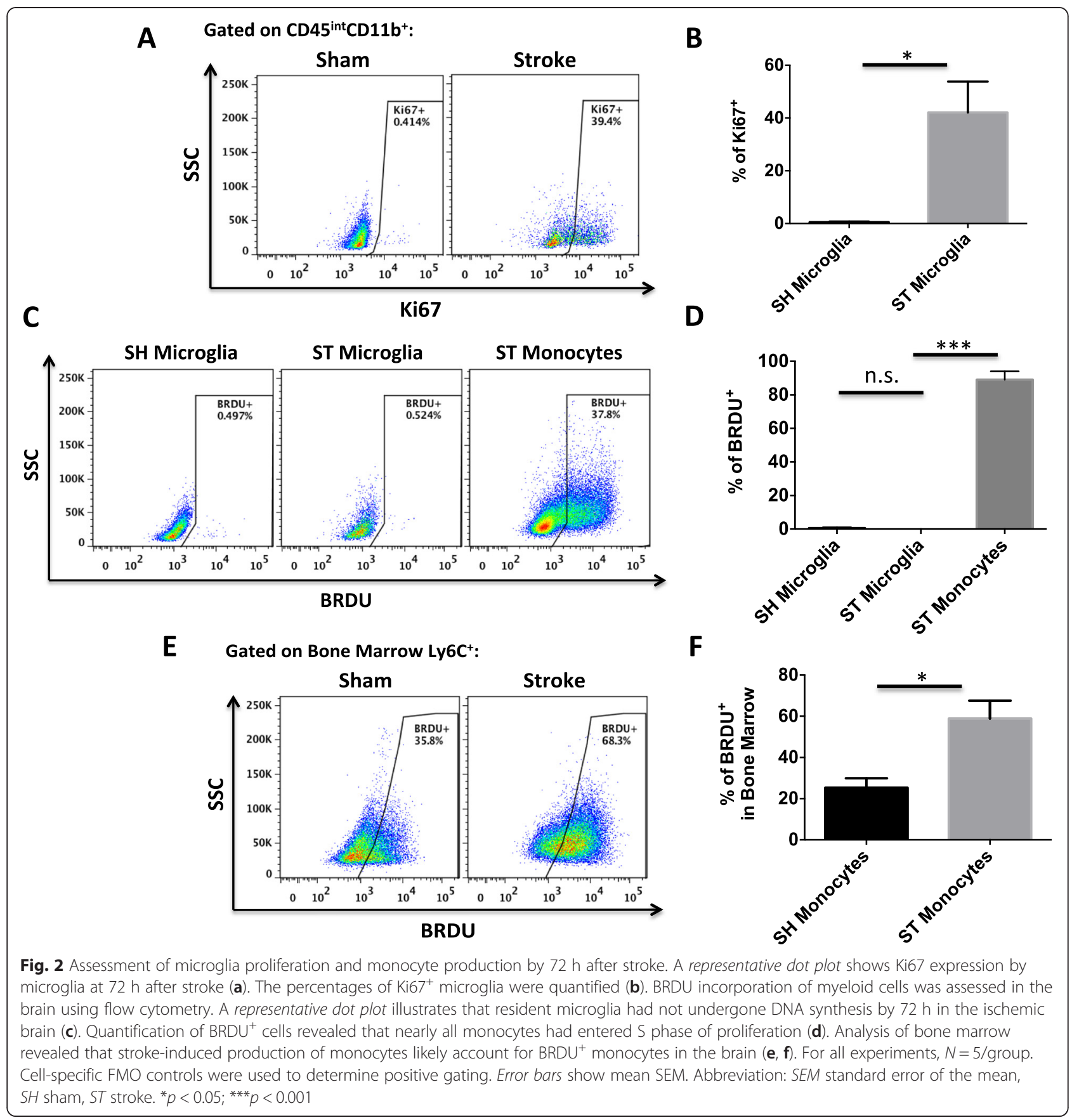

in general appears to be severely compromised after stroke. It is not known whether the microglial population replenishes itself during recovery or if invading monocytes establish permanent residence and take over the microglial niche $[19,20]$.

Although microglia and monocytes exhibit several features of pro-inflammatory activation after stroke, several studies suggest a supportive and even beneficial role for each in stroke outcome [21-27]. These outcomes are likely influenced by the degree of ischemic severity and the ischemic setting/conditions. Distinguishing brain- resident microglia from monocyte populations in ischemic brains that have high numbers of infiltrating leukocytes can be challenging. Due to the lack of available microglia-specific markers, the absolute identification of microglia can be hampered by up-regulation of CD45 expression on activated microglia after injury, which can cause overlap with bone marrow-derived macrophage populations [28-30]. Bone marrow chimeras allowed us to differentiate between all fluorescently tagged bone marrow-derived leukocytes from resident microglia, definitively confirming that microglial expression of CD45 


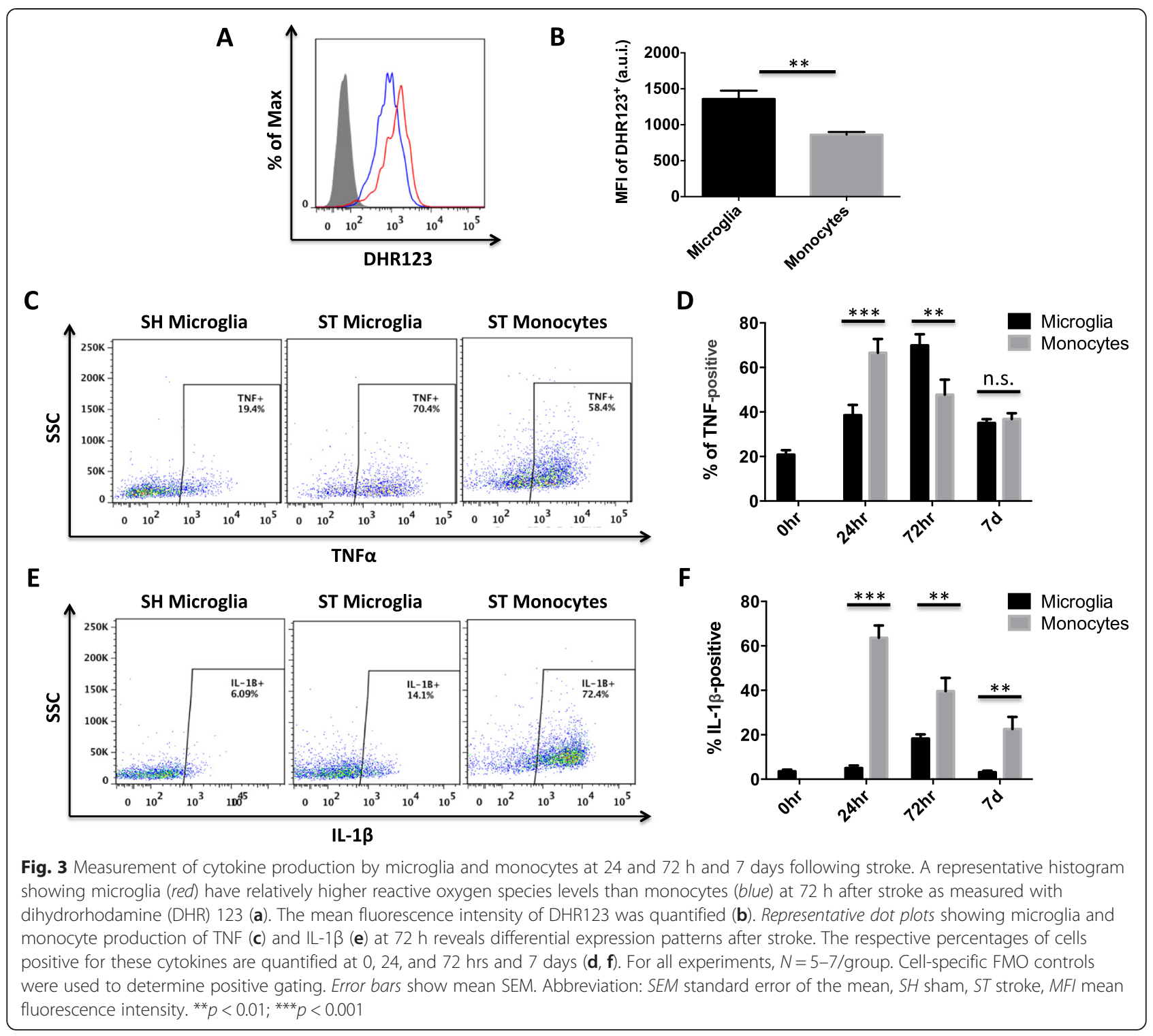

is relatively stable after stroke. However, to further ensure the purity of our microglial gating strategy, we selected out any contaminating monocytes using Ly6C antibody, which is not expressed on adult microglia. These findings enabled us to provide a reliable functional assessment of resident microglia and recruited monocyte populations by ex-vivo flow cytometry.

Recent work from the Merad laboratory has elegantly demonstrated that there is little bone marrow or systemic contribution to the resident microglia pool under normal conditions [31, 32]. These observations were made using donor-tagged bone marrow chimeras and parabiotic mice. Consistent with these findings, our group has also found that as far out as 1 year following radiation, uninjured chimeras had less than $5 \%$ "microglia-like" monocytes as evidenced by their $\mathrm{CD} 45^{\text {int }} \mathrm{CD} 11 \mathrm{~b}^{+} \mathrm{GFP}^{+} \mathrm{Ly} 6 \mathrm{C}^{+}$phenotype, reminiscent of the resident $\mathrm{CD} 45^{\mathrm{int}} \mathrm{CD} 11 \mathrm{~b}^{+} \mathrm{GFP}^{-}$ microglia population (data not shown). Although these findings imply there is a low frequency of monocyte mixing in the resident $\mathrm{CD} 45^{\text {int }}$ microglia population of irradiated chimeras, these bone marrow-derived $\mathrm{GFP}^{+}$cells can still be distinguished by the expression of Ly6C. Although useful, chimera development and parabiotic manipulation have limitations, including the potential for radiation injury and immune activation from the invasive surgical stress, respectively. Importantly, other studies have found that following an injury to the CNS, recruited monocytes can down-regulate Ly6C expression and establish permanent residence, thereby replenishing the attenuated microglia pool [19, 33]. The acute time points described in our study are unlikely to be affected by the down-regulation of Ly6C on monocytes as earlier studies have shown that this does not occur until after 7 days post-injury. While the genotoxic elimination of the microglia in mice appears to 
A

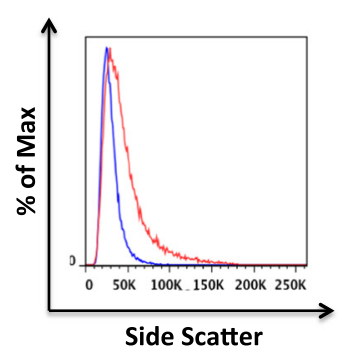

C

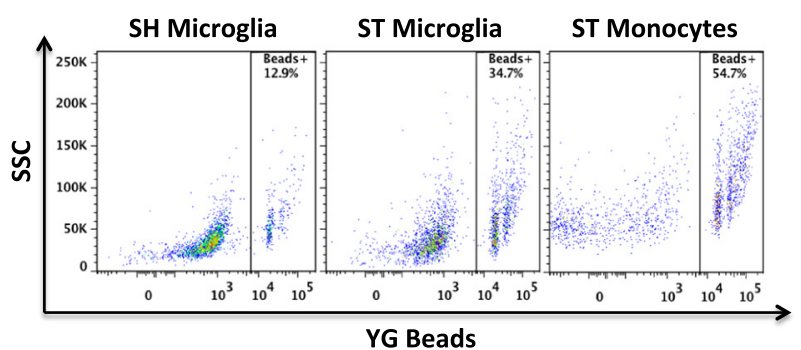

YG Beads

E

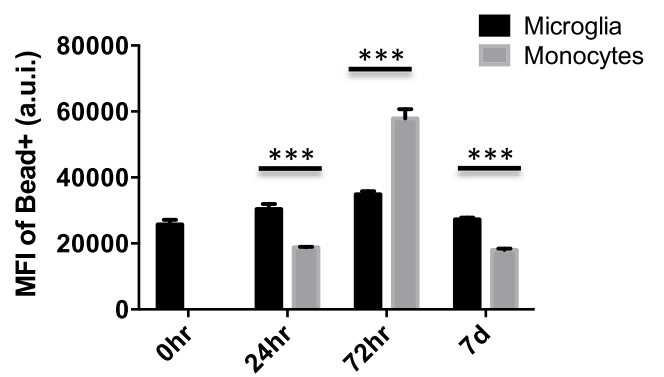

B

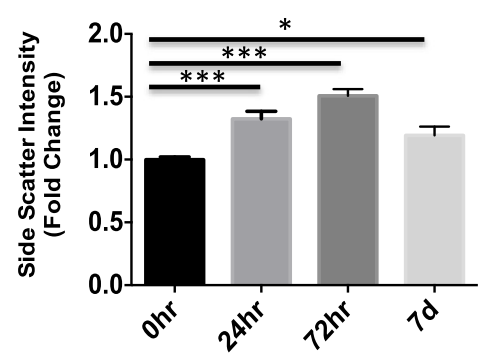

D

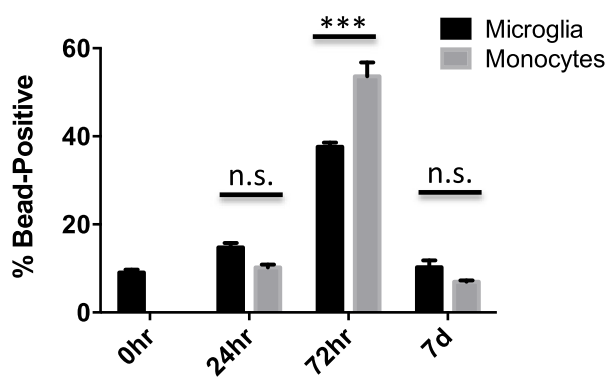

G
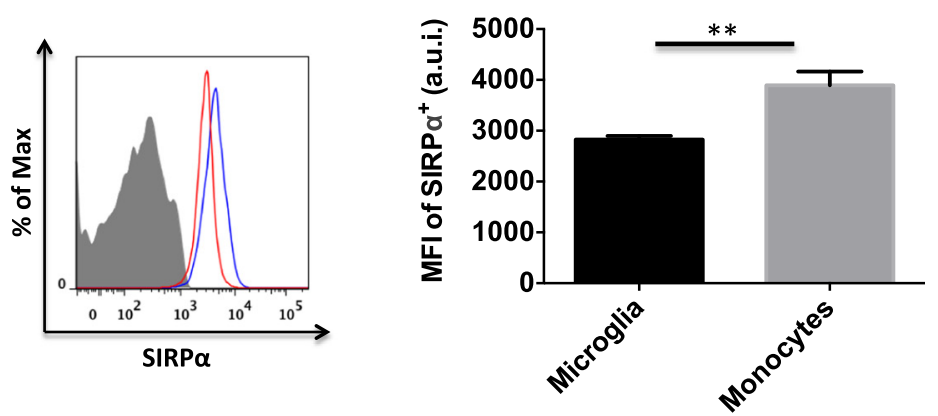

Fig. 4 Phagocytic activity of microglia and monocytes at 24 and $72 \mathrm{~h}$ and 7 days following stroke. Representative histogram showing a relative increase in side scatter (granularity) properties of microglia at $72 \mathrm{~h}$ after stroke (red) compared to sham (blue; a). Mean side scatter intensity of microglia was quantified at different time points after MCAO ( $N=5 /$ group; b). Phagocytic activity after stroke was measured by bead assay using flow cytometry (c). The percentages and mean fluorescence intensity (MFI) of bead ${ }^{+}$cells were quantified at 0, 24, and $72 \mathrm{~h}$ and $7 \mathrm{days}(\mathrm{N}=6$ / group; d, e). A representative histogram shows higher expression of the phagocytic marker SIRPa on monocytes (blue) compared to microglia (red) at $72 \mathrm{~h}$ in the ischemic hemisphere (f). The MFI of SIRPa ${ }^{+}$cells are quantified ( $\left.N=5 / g r o u p ; ~ g\right)$. Cell-specific FMO controls were used to determine positive gating. Error bars show mean SEM. Abbreviation: SEM standard error of the mean, SH sham, ST stroke, SSC side scatter, YG yellow green, a.u.i. arbitrary units of intensity

be non-deleterious in the short-term, the long-term consequences of microglia removal are likely detrimental to CNS homeostasis as evidenced in several neurodegenerative models [34-37]. The long-term consequences of microglia elimination in necrotic regions following ischemic stroke warrants further investigation.

We show that microglia numbers are decreased at $72 \mathrm{~h}$ after a 90-min tMCAO and noted an inverse correlation between microglia and infiltrating monocyte counts. This is in contrast to several reports that have shown significant increases in microglia proliferation [38-40]. However, it is likely that the severity of the ischemic injury plays a major role in the microglial response as other work has shown reduced microglial populations with more severe injury (e.g., longer occlusion times) [41]. Previous work has shown that the morphological changes that accompany glial cell activation after stroke are not dependent on or coincident with neuronal death but rather the duration of ischemia [42]. Microglia are more ischemia-resistant than neurons and oligodendrocytes, but clearly, they are also vulnerable to severe ischemia [43-45]. The inability to distinguish microglia from infiltrating monocytes, which increase in number with injury severity using histological methods, may further obfuscate this issue. Flow cytometry may provide a more sensitive means of identification of cell type based on the well-accepted relative surface expression of CD45. Depending on the compensation setup, overlapping of these cell populations may also occur with flow cytometry. However, we have shown that it is extremely useful to 

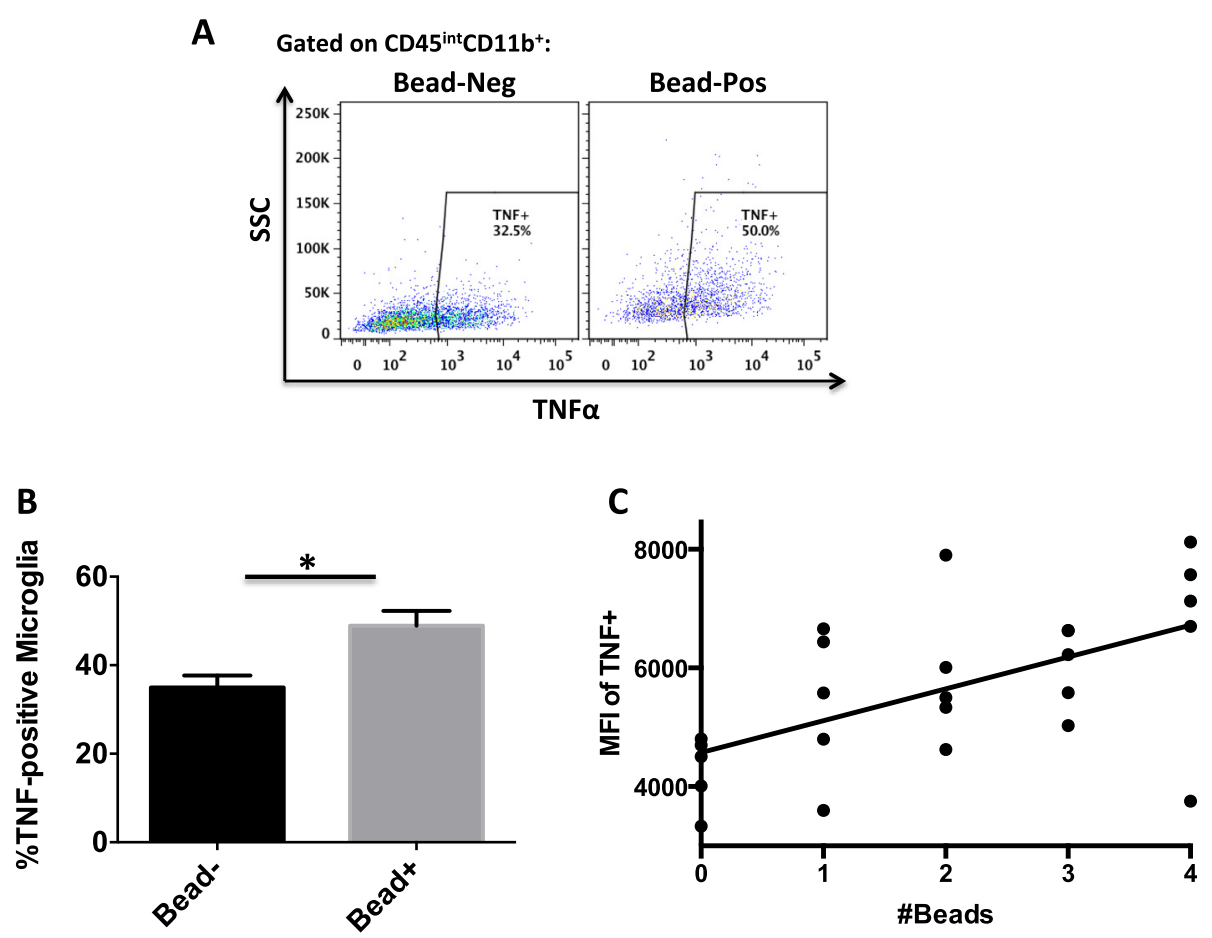

Fig. 5 Relationship between TNF production and phagocytic activity of microglia after stroke. A representative dot plot shows the relationship between microglial TNF production and phagocytic activity at $24 \mathrm{~h} \mathrm{(a)}$. The percentage of TNF${ }^{+}$microglia is significantly higher in the phagocytic subset of microglia compared to the non-phagocytic subset ( $N=5 /$ group; b). A positive correlation was found between the MFI of TNF expression in microglia and number of beads they phagocytosed after stroke (c). Cell-specific FMO controls were used to determine positive gating. Error bars show mean SEM. Abbreviation: SEM standard error of the mean, SSC side scatter, MFI mean fluorescence intensity, a.u.i. arbitrary units of intensity. ${ }^{*} p<0.05$

use Ly6C as a monocyte marker to achieve maximal separation of these two populations, as infiltrating monocytes may down-regulate CD45 and MHCII levels over time, adopting a microglia-like phenotype $\left(\mathrm{CD} 45^{\text {int }} \mathrm{CD} 11 \mathrm{~b}^{+}\right)[33]$.

Increased production of bone marrow-derived myeloid cells after stroke has been described [17]. We found that BRDU is incorporated into many newly produced monocytes in the bone marrow, which are subsequently recruited into the brain. This is consistent with recent work showing ischemic stroke activates hematopoietic stem cells in the bone marrow, resulting in a myeloid bias and greater output of monocytes [46]. The number of BRDU-positive myeloid cells in the brain reaches its peak by $72 \mathrm{~h}[38,41]$. This parallels the time course of leukocyte infiltration into the brain, which also peaks at $72 \mathrm{~h}$. Although microglia did not enter $\mathrm{S}$ phase in our study, many were $\mathrm{Ki} 67^{+}$, indicating that they were actively cycling at $72 \mathrm{~h}$. Ki67 expression in activated microglia after stroke has also been documented [47]. The failure of most cells to progress through later stages of the cell cycle may also be due to altered length of G1 and/or cellular stress [48]. Based on this earlier work and given the differential effects of ischemia on microglia populations located throughout the hemisphere, we hypothesized that some of these cells would enter the $\mathrm{S}$ phase of cell cycle to begin dividing prior to $72 \mathrm{~h}$. Surprisingly, we did not observe this trend. An earlier study using GFP bone marrow chimeras revealed that the vast majority of macrophages in the infarct area after a 30-min TMCAO were derived from local microglia [49]. Despite the smaller injuries and altered leukocyte kinetics that result from shorter occlusion times, the ability to assess microglia numbers after stroke in an unbiased fashion using immunohistochemistry can be challenging due to the subtle difficulty of identifying microglia in their ramified state relative to those with amoeboid morphology and given the rapid migration of activated microglia into the penumbral region often present in the field of view. As such, shifts in population densities due to migration towards injury sites could be misinterpreted as local microglia proliferation. It should be noted, however, that our data do not exclude the possibility that microglia proliferation occurs beyond $72 \mathrm{~h}$ in the 90-min tMCAO model.

A recent study of microglia proliferation after stroke was elegantly approached by implementing the parabiosis model using wild type and CX3CR1 ${ }^{\mathrm{GFP} /-}$ mice [50]. The authors concluded that microglia proliferation accounted for the majority of microgliosis observed after stroke, rather than monocyte infiltration. However, there are several 
possible explanations for the disparate results. The CX3CR1-GFP signal in these reporter mice is not specific only to microglia, especially following an injury stimulus, as it has been shown that Ly6C $\mathrm{C}^{\mathrm{lo}}$ monocytes also express GFP $[30,51,52]$. As histological analyses would not be able to distinguish $\mathrm{GFP}^{+}$microglia from $\mathrm{GFP}^{+}$monocytes (or vice versa in non-GFP WT mice), these cells largely go undetected as they typically begin migrating to the brain within $12-24 \mathrm{~h}$ of injury. In addition, this study used a smaller, more localized injury induced photothrombosis, resulting in a milder inflammatory response. The stimulusdriven production of monocytes in bone marrow in this milder injury would likely be less and further diluted following egress into the blood due to the $\sim 50 \%$ equilibrium of circulating cells in parabiotic (GFP-WT) mice. These studies provide valuable information in the context of both the specific model of stroke being employed, the severity of ischemia, and the time points being investigated. Taken together, these authors nicely demonstrate that microglia proliferation occurs early after stroke under mild or modest ischemic conditions, whereas our data implies this function is likely compromised after severe ischemic injury.

Oxidative stress and cytokine-induced cell death are key components of ischemic injury. Reperfusion after ischemia produces a burst in ROS formation [53]. Oxidative stress increases within the first hour after reperfusion and delivers signals that promote necrosis and apoptosis. The generation of ROS burst activity results from impaired mitochondrial respiratory chain function and activation of cytoplasmic oxidases. As a result, oxidative stress contributes directly to necrosis and apoptosis through a number of pathways in ischemic tissue. Although neurons and endothelial cells are important sources of ROS, all cells subject to ischemic injury are vulnerable to ROS-mediated cell death signaling [54]. The massive influx of ROS producing infiltrating leukocytes adds considerably to the overall ROS levels in the brain after stroke. Using bone marrow chimeras, a recent study demonstrated that $\mathrm{NADPH}$ oxidase (NOX2)-mediated ROS production in circulating leukocytes contributed to exacerbated infarct volumes compared to that produced by resident microglia after stroke [55]. Although we found that ROS levels were higher in microglia after stroke, we did not evaluate the other subsets of infiltrating myeloid cells, namely neutrophils. Instead, we show that on cell-to-cell basis, monocytes produce significantly less ROS after stroke than resident microglia. This may reflect the severely compromised state of microglia following ischemic reperfusion.

Injury-driven production of ROS can further amplify the inflammatory response by driving cytokine production. TNF and IL- $1 \beta$ are produced by both microglia and monocytes after stroke. Numerous studies using transgenic mice and pharmacological agents have demonstrated a neurotoxic role for each of these pro-inflammatory cytokines in ischemic injury $[56,57]$. Our finding that microglia produced higher levels of ROS and TNF after stroke than monocytes suggest that the resident cells may be the more detrimental macrophage early after injury. Excessive insult to resident microglia may impair their primary function to maintain brain homeostasis during recovery. These findings build on previous work which described the expression of these cytokines in different subsets of microglia and macrophages $24 \mathrm{~h}$ after permanent MCAO using a bone marrow chimera approach combined with immunohistochemistry and flow cytometry [58]. The authors reported that a greater number of $\mathrm{CD} 11 \mathrm{~b}^{+} \mathrm{CD} 45^{\mathrm{hi}}$ macrophages/ granulocytes expressed TNF and IL-1 $\beta$ at $24 \mathrm{~h}$ compared to $\mathrm{CD} 11 \mathrm{~b}^{+} \mathrm{CD} 45^{\mathrm{dim}}$ microglia. While innovative in its design, the use of GR-1 to distinguish between and identify monocytes/macrophages and neutrophils is not as accurate as Ly6C/Ly6G markers [51]. Despite this discrepancy, we found similar expression profiles in these two populations at $24 \mathrm{~h}$, whereas microglia became the predominant TNF producers by peak leukocyte infiltration at $72 \mathrm{~h}$. The importance of TNF signaling to ischemic injury was recently evaluated [59]. Using bone marrow chimeras, it was found that TLR2- and TLR4-mediated injury is primarily driven by infiltrating leukocytes rather than resident microglia. However, TNF is also produced by microglia early after ischemia prior to monocyte infiltration, and TNF-directed therapies at these early time points are neuroprotective $[60,61]$. However, it must also be noted that infiltrating monocytes expressed higher levels of IL-1 $\beta$, a cytokine associated with inflammasome signaling [62, 63]. This implies that inflammatory signaling in macrophage populations is incredibly robust and complex. Further understanding of these differences between these two cell subtypes and their inflammatory function after stroke may provide better insight on the targeted effects of known drug treatments, allowing for the development of more effective, cell-specific drugs.

Following ischemia, injured areas of the brain undergo apoptosis. Dead and dying cells generate a vast amount of debris that requires clearance for regenerative processes to ensue. As the ischemic/reperfusion injury core evolves after $24 \mathrm{~h}$, amoeboid microglia migrate from the core to the transition zone of the penumbral area following reperfusion and adopt an M1 phenotype [64, 65]. The importance of debris clearance by phagocytes is evident in rodents and humans lacking the scavenger receptor gene CD36, resulting in exacerbated injury and hematoma absorption in models of ICH [66, 67]. MRI imaging has demonstrated that USPIO particles can be tracked and localized to ED1+ microglia/macrophages with the highest signal intensities found in striatal and cortical penumbral regions at day 2 after 30 min tMCAO in rats [68]. Using GFP bone marrow chimeras, an earlier 
study had shown that activated microglia phagocytose neuronal material as early as $24 \mathrm{~h}$ and are the predominant phagocyte by day 7 [69]. While an early preponderance of phagocytic microglia would be expected given the delayed recruitment of monocytes, their use of a less severe, 30-min tMCA occlusion resulted in attenuated numbers of infiltrating monocytes. In another study, rats that had undergone a 3-hr tMCAO exhibited poor microglial phagocytosis of caspase-3-expressing neurons at $24 \mathrm{~h}$ [24]. Moreover, the number of apoptotic neurons was not increased following liposome-mediated ablation of microglia, indicating a limited contribution to debris clearance in environments that are significantly impacted by ischemia. Injury-related defects in migration and increased cytokine repulsion signals may halt microglia phagocytic activity under increasing ischemic stress.

We provide the first functional evidence that microglia increase capacity for phagocytosis in acute ischemic stroke. The number of phagocytic microglia initially increased at $24 \mathrm{~h}$, peaked at $72 \mathrm{~h}$, and returned to baseline by 7 days. These data paralleled the increase in microglia side scatter properties (granularity) in separate experiments, further indication of phagocytic uptake. Interestingly, however, infiltrating monocytes were significantly more efficient at phagocytosing material during the peak response at $72 \mathrm{~h}$. Following tissue entry, monocytes may further mature into macrophage or dendritic cell subsets with tissue-specialized functions. Monocyte-derivatives retain the expression of lineage markers and have enhanced phagocytic ability [70]. Our findings indicate that while these tissue phagocytes exhibit many classical pro-inflammatory features deemed destructive, they appear to play a critical role in debris clearance and post-stroke recovery. It may be that in addition to being injured, many resident microglia had already exhausted their capacity to phagocytize surplus debris prior to ex-vivo phagocytosis assessment. However, by 7 days after stroke, when monocytes diminish in the ischemic brain, resident microglia again become the predominant phagocyte, highlighting the importance of microglia in the long-term recovery and repair process. Unpublished results from our laboratory suggest that circulating phagocyte populations are generally more efficient than microglia at phagocytizing material under non-stimulated conditions. Recent work demonstrating the importance of monocytes to hemorrhage clearance in a model of intracerebral hemorrhage highlights their ability to significantly improve long-term outcomes through phagocytic function early after stroke [71]. This may relate in part to the degree of adaptive functionality associated with their different life-spans, as monocyte function may be more robust given their "boom and bust" half-life, whereas the phagocytic activity of microglia likely endures at a steady rate throughout life. It is important to note that monocyte-targeted therapies aimed at inhibiting the function of these cells may inadvertently affect their phagocytic activity. Moreover, strategies to exploit this capacity should be considered, especially during the early stages of injury when the blood-brain barrier is susceptible to monocyte entry.

Finally, we showed a positive correlation between TNF expression levels and the level of phagocytic activity of microglia after injury. ROS and pro-inflammatory cytokine signaling is thought to hamper phagocytic activity and debris clearance $[72,73]$. Our finding suggests that dichotomizing function into M1/M2 polarization, in which cells are polarized to become either pro-inflammatory/ detrimental (M1) or anti-inflammatory/injury resolving (M2) may be too simplistic [74]. This concept more aptly applies to microglia in vitro and its polarization may be better appreciated as a continuum of activation in vivo due to the combination of factors and diverse cell types present in intact tissue. Indeed, the polarization dynamics of microglia and monocytes during stroke appear to be mixed and complex. As mentioned, anti-inflammatory markers such as IL-4 and IL-10 were not expressed by either myeloid cell at any time point in this study. However, we acknowledge that transitioning between M1 and M2 phases may occur weeks after injury as has been reported by others $[5,65]$. Whether there is a requirement for proinflammatory signaling to induce or enhance phagocytic activity and debris clearance is currently not known. These findings point to a possible side effect of microglia/ macrophage inhibition that could hamper the removal of dead cells and slow recovery.

In conclusion, we have identified distinct functional roles for brain-resident and infiltrating macrophages after stroke. To better both the interpretation of failed clinical trials and the development of more efficacious drugs to treat sterile inflammation in the CNS requires a better understanding of potential cellular targets. We believe that functional assessment of immune cells after stroke ex-vivo could be a powerful tool in screening and validating the targeted cellular effects of drugs and various therapeutic interventions.

\section{Additional file}

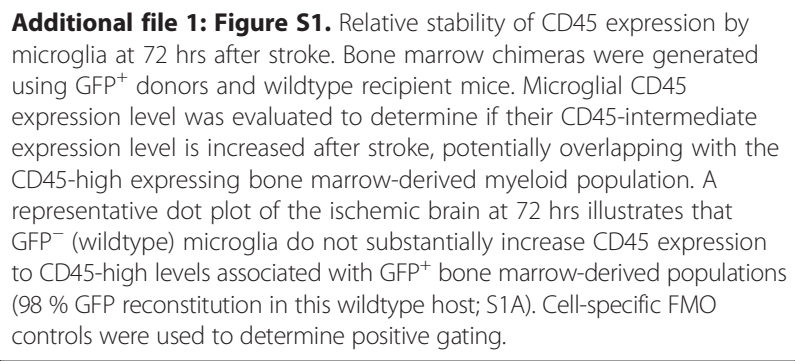

Abbreviations

BRDU: bromodeoxyuridine; CNS: central nervous system;

DHR123: dihydrorhodamine 123; FMO: fluorescence minus one; GFP: green 
fluorescent protein; L-1 3 : interleukin-1 beta; MFI: mean fluorescence intensity; ROS: reactive oxygen species; $\mathrm{SH}$ : sham surgery; SIRPa: signal-regulatory protein alpha; SSC: side scatter; ST: stroke surgery; TNF: tumor necrosis factor; tMCAO: transient middle cerebral artery occlusion.

\section{Competing interests}

The authors declare that they have no competing interests.

\section{Authors' contributions}

RMR and LDM conceived the project. RMR performed most of the experiments and ARP, JMG, and JC assisted with the flow cytometry preparations. RMR and ERJ performed FACS analysis and interpretation. RV assisted with the statistical analyses. RMR and LDM wrote the paper. All authors read and made comments on the manuscript draft and approved the final manuscript.

\section{Acknowledgements}

This work was supported by National Institutes of Health grants R21 NS082906-02 (Louise D. McCullough), R21 NS076293-01A1 (Louise D. McCullough), and F31 NS083244-01A1 (Rodney M. Ritzel).

\section{Author details}

'Department of Neurology, University of Connecticut Health Center, 263 Farmington Avenue, Farmington, CT 06030, USA. ${ }^{2}$ Department of Immunology, University of Connecticut Health Center, Farmington, CT, USA.

\section{Received: 3 March 2015 Accepted: 20 May 2015}

Published online: 29 May 2015

\section{References}

1. Whiteley W, Jackson C, Lewis S, Lowe G, Rumley A, Sandercock P, et al. Inflammatory markers and poor outcome after stroke: a prospective cohort study and systematic review of interleukin-6. PLoS Med. 2009;6. e1000145.

2. Di Napoli M, Papa F, Bocola V. C-reactive protein in ischemic stroke: an independent prognostic factor. Stroke. 2001;32:917-24.

3. Vila N, Castillo J, Davalos A, Esteve A, Planas AM, Chamorro A. Levels of anti-inflammatory cytokines and neurological worsening in acute ischemic stroke. Stroke. 2003;34:671-5.

4. Vila N, Castillo J, Davalos A, Chamorro A. Proinflammatory cytokines and early neurological worsening in ischemic stroke. Stroke. 2000;31:2325-9.

5. Benakis C, Garcia-Bonilla L, ladecola C, Anrather J. The role of microglia and myeloid immune cells in acute cerebral ischemia. Front Cell Neurosci. 2014;8:461.

6. Joseph B, Venero JL. A brief overview of multitalented microglia. Methods Mol Biol. 2013;1041:3-8.

7. Patel AR, Ritzel R, McCullough LD, Liu F. Microglia and ischemic stroke: a double-edged sword. Int J Physiol Pathophysiol Pharmacol. 2013;5:73-90.

8. London A, Cohen M, Schwartz M. Microglia and monocyte-derived macrophages: functionally distinct populations that act in concert in CNS plasticity and repair. Front Cell Neurosci. 2013;7:34.

9. Prinz M, Priller J, Sisodia SS, Ransohoff RM. Heterogeneity of CNS myeloid cells and their roles in neurodegeneration. Nat Neurosci. 2011;14:1227-35.

10. Mildner A, Schlevogt B, Kierdorf K, Bottcher C, Erny D, Kummer MP, et al. Distinct and non-redundant roles of microglia and myeloid subsets in mouse models of Alzheimer's disease. J Neurosci. 2011;31:11159-71.

11. Yamasaki R, Lu H, Butovsky O, Ohno N, Rietsch AM, Cialic R, et al. Differential roles of microglia and monocytes in the inflamed central nervous system. J Exp Med. 2014;211:1533-49.

12. Tang $X N$, Zheng Z, Yenari MA. Bone marrow chimeras in the study of experimental stroke. Transl Stroke Res. 2012;3:341-7.

13. McCullough LD, Zeng Z, Blizzard KK, Debchoudhury I, Hurn PD. Ischemic nitric oxide and poly (ADP-ribose) polymerase-1 in cerebral ischemia: male toxicity, female protection. J Cereb Blood Flow Metab. 2005;25:502-12.

14. Voss EV, Skuljec J, Gudi V, Skripuletz T, Pul R, Trebst C, et al. Characterisation of microglia during de- and remyelination: can they create a repair promoting environment? Neurobiol Dis. 2012;45:519-28.

15. Pul R, Chittappen KP, Stangel M. Quantification of microglial phagocytosis by a flow cytometer-based assay. Methods Mol Biol. 2013;1041:121-7.

16. Schaefer BC, Schaefer ML, Kappler JW, Marrack P, Kedl RM. Observation of antigen-dependent CD8+ T-cell/ dendritic cell interactions in vivo. Cell Immunol. 2001;214:110-22.
17. Denes A, McColl BW, Leow-Dyke SF, Chapman KZ, Humphreys NE, Grencis RK, et al. Experimental stroke-induced changes in the bone marrow reveal complex regulation of leukocyte responses. J Cereb Blood Flow Metab. 2011;31:1036-50.

18. Chan PH. Reactive oxygen radicals in signaling and damage in the ischemic brain. J Cereb Blood Flow Metab. 2001;21:2-14.

19. Varvel NH, Grathwohl SA, Baumann F, Liebig C, Bosch A, Brawek B, et al. Microglial repopulation model reveals a robust homeostatic process for replacing CNS myeloid cells. Proc Natl Acad Sci U S A. 2012;109:18150-5.

20. Mildner A, Schmidt H, Nitsche M, Merkler D, Hanisch UK, Mack M, et al. Microglia in the adult brain arise from Ly-6ChiCCR2+ monocytes only under defined host conditions. Nat Neurosci. 2007;10:1544-53.

21. Chiba T, Umegaki K. Pivotal roles of monocytes/macrophages in stroke. Mediators Inflamm. 2013;2013:759103.

22. Womble TA, Green S, Shahaduzzaman M, Grieco J, Sanberg PR, Pennypacker KR, et al. Monocytes are essential for the neuroprotective effect of human cord blood cells following middle cerebral artery occlusion in rat. Mol Cell Neurosci. 2014;59:76-84.

23. Schilling M, Strecker JK, Ringelstein EB, Schabitz WR, Kiefer R. The role of CC chemokine receptor 2 on microglia activation and blood-borne cell recruitment after transient focal cerebral ischemia in mice. Brain Res. 2009;1289:79-84.

24. Faustino JV, Wang X, Johnson CE, Klibanov A, Derugin N, Wendland MF, et al. Microglial cells contribute to endogenous brain defenses after acute neonatal focal stroke. J Neurosci. 2011;31:12992-3001.

25. Lalancette-Hebert M, Gowing G, Simard A, Weng YC, Kriz J. Selective ablation of proliferating microglial cells exacerbates ischemic injury in the brain. J Neurosci. 2007;27:2596-605.

26. Schuette-Nuetgen $K$, Strecker JK, Minnerup J, Ringelstein EB, Schilling M. MCP-1/CCR-2-double-deficiency severely impairs the migration of hematogenous inflammatory cells following transient cerebral ischemia in mice. Exp Neurol. 2012;233:849-58.

27. Hughes PM, Allegrini PR, Rudin M, Perry VH, Mir AK, Wiessner C. Monocyte chemoattractant protein-1 deficiency is protective in a murine stroke model. J Cereb Blood Flow Metab. 2002;22:308-17.

28. Ponomarev ED, Shriver LP, Maresz K, Dittel BN. Microglial cell activation and proliferation precedes the onset of CNS autoimmunity. J Neurosci Res. 2005;81:374-89.

29. Carson MJ, Bilousova TV, Puntambekar SS, Melchior B, Doose JM, Ethell IM. A rose by any other name? The potential consequences of microglial heterogeneity during CNS health and disease. Neurotherapeutics. 2007;4:571-9.

30. Butovsky O, Jedrychowski MP, Moore CS, Cialic R, Lanser AJ, Gabriely G, et al. Identification of a unique TGF-beta-dependent molecular and functional signature in microglia. Nat Neurosci. 2014;17:131-43.

31. Ginhoux F, Greter M, Leboeuf M, Nandi S, See P, Gokhan S, et al. Fate mapping analysis reveals that adult microglia derive from primitive macrophages. Science. 2010;330:841-5.

32. Hashimoto D, Chow A, Noizat C, Teo P, Beasley MB, Leboeuf M, et al. Tissue-resident macrophages self-maintain locally throughout adult life with minimal contribution from circulating monocytes. Immunity. 2013;38:792-804

33. Getts DR, Terry RL, Getts MT, Muller M, Rana S, Shrestha B, et al. Ly6c + "inflammatory monocytes" are microglial precursors recruited in a pathogenic manner in West Nile virus encephalitis. J Exp Med. 2008;205:2319-37.

34. Grathwohl SA, Kalin RE, Bolmont T, Prokop S, Winkelmann G, Kaeser SA, et al. Formation and maintenance of Alzheimer's disease beta-amyloid plaques in the absence of microglia. Nat Neurosci. 2009;12:1361-3.

35. Derecki NC, Cronk JC, Lu Z, Xu E, Abbott SB, Guyenet PG, et al. Wild-type microglia arrest pathology in a mouse model of Rett syndrome. Nature. 2012;484:105-9.

36. Chouery E, Delague V, Bergougnoux A, Koussa S, Serre JL, Megarbane A. Mutations in TREM2 lead to pure early-onset dementia without bone cysts. Hum Mutat. 2008;29:E194-204.

37. Neumann $\mathrm{H}$, Takahashi K. Essential role of the microglial triggering receptor expressed on myeloid cells-2 (TREM2) for central nervous tissue immune homeostasis. J Neuroimmunol. 2007:184:92-9.

38. Bohacek I, Cordeau P, Lalancette-Hebert M, Gorup D, Weng YC, Gajovic S, et al. Toll-like receptor 2 deficiency leads to delayed exacerbation of ischemic injury. J Neuroinflammation. 2012;9:191.

39. Jin R, Yang G, Li G. Inflammatory mechanisms in ischemic stroke: role of inflammatory cells. J Leukoc Biol. 2010;87:779-89. 
40. Baba T, Kameda M, Yasuhara T, Morimoto T, Kondo A, Shingo T, et al. Electrical stimulation of the cerebral cortex exerts antiapoptotic, angiogenic, and anti-inflammatory effects in ischemic stroke rats through phosphoinositide 3-kinase/Akt signaling pathway. Stroke. 2009;40:e598-605.

41. Denes A, Vidyasagar R, Feng J, Narvainen J, McColl BW, Kauppinen RA, et al. Proliferating resident microglia after focal cerebral ischaemia in mice. J Cereb Blood Flow Metab. 2007;27:1941-53.

42. Sugawara T, Lewen A, Noshita N, Gasche Y, Chan PH. Effects of global ischemia duration on neuronal, astroglial, oligodendroglial, and microglial reactions in the vulnerable hippocampal CA1 subregion in rats. J Neurotrauma. 2002;19:85-98.

43. Yenari MA, Giffard RG. Ischemic vulnerability of primary murine microglial cultures. Neurosci Lett. 2001;298:5-8.

44. Panickar KS, Norenberg MD. Astrocytes in cerebral ischemic injury: morphological and general considerations. Glia. 2005;50:287-98.

45. Dewar D, Underhill SM, Goldberg MP. Oligodendrocytes and ischemic brain injury. J Cereb Blood Flow Metab. 2003;23:263-74.

46. Courties G, Herisson F, Sager HB, Heidt T, Ye Y, Wei Y, et al. Ischemic stroke activates hematopoietic bone marrow stem cells. Circ Res. 2015;116:407-17.

47. Zhang Q, Chen C, Lu J, Xie M, Pan D, Luo X, et al. Cell cycle inhibition attenuates microglial proliferation and production of IL-1beta, MIP-1alpha, and NO after focal cerebral ischemia in the rat. Glia. 2009;57:908-20.

48. Ishimura R, Martin GR, Ackerman SL. Loss of apoptosis-inducing factor results in cell-type-specific neurogenesis defects. J Neurosci. 2008;28:4938-48.

49. Schilling $M$, Besselmann M, Leonhard C, Mueller M, Ringelstein EB, Kiefer R. Microglial activation precedes and predominates over macrophage infiltration in transient focal cerebral ischemia: a study in green fluorescent protein transgenic bone marrow chimeric mice. Exp Neurol. 2003;183:25-33.

50. Li T, Pang S, Yu Y, Wu X, Guo J, Zhang S. Proliferation of parenchymal microglia is the main source of microgliosis after ischaemic stroke. Brain. 2013;136:3578-88.

51. Rose S, Misharin A, Perlman H. A novel Ly6C/Ly6G-based strategy to analyze the mouse splenic myeloid compartment. Cytometry A. 2012;81:343-50.

52. Butovsky O, Siddiqui S, Gabriely G, Lanser AJ, Dake B, Murugaiyan G, et al. Modulating inflammatory monocytes with a unique microRNA gene signature ameliorates murine ALS. J Clin Invest. 2012;122:3063-87.

53. Peters $O$, Back T, Lindauer U, Busch C, Megow D, Dreier J, et al. Increased formation of reactive oxygen species after permanent and reversible middle cerebral artery occlusion in the rat. J Cereb Blood Flow Metab. 1998;18:196-205.

54. Manzanero S, Santro T, Arumugam TV. Neuronal oxidative stress in acute ischemic stroke: sources and contribution to cell injury. Neurochem Int. 2013;62:712-8.

55. Tang XN, Zheng Z, Giffard RG, Yenari MA. Significance of marrow-derived nicotinamide adenine dinucleotide phosphate oxidase in experimental ischemic stroke. Ann Neurol. 2011;70:606-15.

56. Lambertsen KL, Biber K, Finsen B. Inflammatory cytokines in experimental and human stroke. J Cereb Blood Flow Metab. 2012;32:1677-98.

57. Moskowitz MA, Lo EH, ladecola C. The science of stroke: mechanisms in search of treatments. Neuron. 2010;67:181-98.

58. Clausen BH, Lambertsen KL, Babcock AA, Holm TH, Dagnaes-Hansen F, Finsen B. Interleukin-1beta and tumor necrosis factor-alpha are expressed by different subsets of microglia and macrophages after ischemic stroke in mice. J Neuroinflammation. 2008:5:46.

59. Shichita T, Hasegawa E, Kimura A, Morita R, Sakaguchi R, Takada I, et al. Peroxiredoxin family proteins are key initiators of post-ischemic inflammation in the brain. Nat Med. 2012;18:911-7.

60. Caso JR, Pradillo JM, Hurtado O, Lorenzo P, Moro MA, Lizasoain I. Toll-like receptor 4 is involved in brain damage and inflammation after experimental stroke. Circulation. 2007;115:1599-608.

61. Sumbria RK, Boado RJ, Pardridge WM. Brain protection from stroke with intravenous TNFalpha decoy receptor-Trojan horse fusion protein. J Cereb Blood Flow Metab. 2012;32:1933-8.

62. Abulafia DP, de Rivero Vaccari JP, Lozano JD, Lotocki G, Keane RW, Dietrich WD. Inhibition of the inflammasome complex reduces the inflammatory response after thromboembolic stroke in mice. J Cereb Blood Flow Metab. 2009:29:534-44.

63. Zhang N, Zhang X, Liu X, Wang H, Xue J, Yu J, et al. Chrysophanol inhibits NALP3 inflammasome activation and ameliorates cerebral ischemia/ reperfusion in mice. Mediators Inflamm. 2014;2014:370530.

64. Tanaka R, Komine-Kobayashi M, Mochizuki H, Yamada M, Furuya T, Migita M, et al. Migration of enhanced green fluorescent protein expressing bone marrow-derived microglia/macrophage into the mouse brain following permanent focal ischemia. Neuroscience. 2003;117:531-9.

65. Hu X, Li P, Guo Y, Wang H, Leak RK, Chen S, et al. Microglia/macrophage polarization dynamics reveal novel mechanism of injury expansion after focal cerebral ischemia. Stroke. 2012;43:3063-70.

66. Woo MS, Wang X, Faustino JV, Derugin N, Wendland MF, Zhou P, et al. Genetic deletion of CD36 enhances injury after acute neonatal stroke. Ann Neurol. 2012;72:961-70.

67. Fang $H$, Chen J, Lin S, Wang P, Wang Y, Xiong X, et al. CD36-mediated hematoma absorption following intracerebral hemorrhage: negative regulation by TLR4 signaling. J Immunol. 2014;192:5984-92.

68. Rausch M, Sauter A, Frohlich J, Neubacher U, Radu EW, Rudin M. Dynamic patterns of USPIO enhancement can be observed in macrophages after ischemic brain damage. Magn Reson Med. 2001;46:1018-22.

69. Schilling M, Besselmann M, Muller M, Strecker JK, Ringelstein EB, Kiefer R. Predominant phagocytic activity of resident microglia over hematogenous macrophages following transient focal cerebral ischemia: an investigation using green fluorescent protein transgenic bone marrow chimeric mice. Exp Neurol. 2005;196:290-7.

70. Geissmann F, Manz MG, Jung S, Sieweke MH, Merad M, Ley K. Development of monocytes, macrophages, and dendritic cells. Science. 2010;327:656-61.

71. Hammond MD, Taylor RA, Mullen MT, Ai Y, Aguila HL, Mack M, et al. CCR2+ Ly6C(hi) inflammatory monocyte recruitment exacerbates acute disability following intracerebral hemorrhage. J Neurosci. 2014;34:3901-9.

72. Mallat M, Marin-Teva JL, Cheret C. Phagocytosis in the developing CNS: more than clearing the corpses. Curr Opin Neurobiol. 2005;15:101-7.

73. Lauber K, Blumenthal SG, Waibel M, Wesselborg S. Clearance of apoptotic cells: getting rid of the corpses. Mol Cell. 2004;14:277-87.

74. Murray PJ, Allen JE, Biswas SK, Fisher EA, Gilroy DW, Goerdt S, et al. Macrophage activation and polarization: nomenclature and experimental guidelines. Immunity. 2014;41:14-20.

\section{Submit your next manuscript to BioMed Central and take full advantage of:}

- Convenient online submission

- Thorough peer review

- No space constraints or color figure charges

- Immediate publication on acceptance

- Inclusion in PubMed, CAS, Scopus and Google Scholar

- Research which is freely available for redistribution 